\title{
Orbital Characteristics of the Subdwarf-B and F V Star Binary EC 20117-4014 (=V4640 Sgr $)$
}

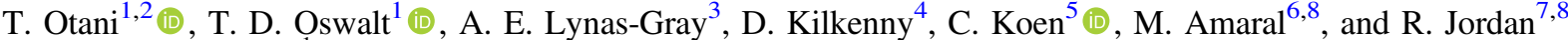 \\ ${ }^{1}$ Department of Physical Sciences and SARA, Embry-Riddle Aeronautical University, \\ 600 S. Clyde Morris Boulevard, Daytona Beach, FL 32114, USA; otanit@erau.edu \\ ${ }^{2}$ Department of Physics \& Space Sciences, Florida Institute of Technology, 150 W University Boulevard, Melbourne, FL 32901, USA \\ ${ }^{3}$ Department of Physics, University of Oxford, Oxford, OX1 3RH, UK \\ ${ }^{4}$ Department of Physics and Astronomy, University of the Western Cape, Bellville 7535, South Africa \\ ${ }^{5}$ Department of Statistics, University of the Western Cape, Bellville 7535, South Africa \\ ${ }^{6}$ Department of Science, Cape Cod Community College, 2240 Iyannough Road, West Barnstable, MA 02668, USA \\ ${ }_{8}^{7}$ Astronomy Department, Whitman College, 345 Boyer Avenue, Walla Walla, WA 99362, USA \\ ${ }^{8}$ Southeastern Association for Research in Astronomy (SARA) NSF-REU Summer Intern (2015) \\ Received 2017 June 26; revised 2018 March 23; accepted 2018 March 23; published 2018 June 1
}

\begin{abstract}
Among the competing evolution theories for subdwarf-B (sdB) stars is the binary evolution scenario. EC 201174014 (=V4640 Sgr) is a spectroscopic binary system consisting of a pulsating sdB star and a late F main-sequence companion; however, the period and the orbit semimajor axes have not been precisely determined. This paper presents orbital characteristics of the EC 20117-4014 binary system using 20 years of photometric data. Periodic observed minus calculated $(O-C)$ variations were detected in the two highest-amplitude pulsations identified in the EC 20117-4014 power spectrum, indicating the binary system's precise orbital period $(P=792.3$ days $)$ and the light-travel-time amplitude $(A=468.9 \mathrm{~s})$. This binary shows no significant orbital eccentricity, and the upper limit of the eccentricity is 0.025 (using $3 \sigma$ as an upper limit). This upper limit of the eccentricity is the lowest among all wide sdB binaries with known orbital parameters. This analysis indicated that the sdB is likely to have lost its hydrogen envelope through stable Roche lobe overflow, thus supporting hypotheses for the origin of sdB stars. In addition to those results, the underlying pulsation period change obtained from the photometric data was $\dot{P}=5.4( \pm 0.7) \times 10^{-14} \mathrm{~d} \mathrm{~d}^{-1}$, which shows that the sdB is just before the end of the core helium-burning phase.
\end{abstract}

Key words: binaries: spectroscopic - stars: evolution - stars: individual (EC 20117-4014) - stars: oscillations subdwarfs

Supporting material: figure sets, machine-readable table

\section{Introduction}

Subdwarf-B (sdB) stars are core helium-burning objects found in both the disk and halo of our Galaxy (Saffer et al. 1994). V361 Hya was the first pulsating sdB star to be discovered (Kilkenny et al. 1997), and Østensen et al. (2010) subsequently discovered only 20 new pulsators among the more than $300 \mathrm{sdBs}$ predicted to lie in the V361 Hya instability strip and monitored, suggesting that only about $10 \%$ of these $\mathrm{sdB}$ stars have pulsations detectable from the ground. The $\mathrm{sdB}$ observed properties place them in the extreme horizontal branch (EHB) part of the $\mathrm{H}-\mathrm{R}$ diagram. Their effective temperatures range from 22,000 to $40,000 \mathrm{~K}$, and surface gravities range from $5.0 \leqslant \log g \leqslant 6.2$ (in cgs units). Their masses are narrowly confined to about $0.5 M_{\odot}$ (Heber 2009). Subdwarf-B stars have experienced mass loss at the end of the red giant branch phase (Bonanno et al. 2003), in which the hydrogen envelope is lost, leaving a helium core with a very thin inert hydrogen-rich envelope. The loss of the hydrogen envelope prevents the star from ascending the asymptotic giant branch, and the star settles on the EHB, spending about $10^{8}$ years as an sdB star. Upon helium depletion in the core, such a star becomes a subdwarf $\mathrm{O}(\mathrm{sdO})$ star, burning helium in a shell surrounding a $\mathrm{C} / \mathrm{O}$ core and, eventually, a DAO white dwarf (Dorman et al. 1993; Bergeron et al. 1994).

The formation mechanism of sdB stars, i.e., why they lose their hydrogen envelopes, is a matter of current debate. Plausible sdB formation models via binary evolution were constructed by Han et al. (2002, 2003). According to Silvotti et al. (2011), companion stars have been detected in at least $50 \%$ of sdB stars, strongly supporting a binary origin. However, some fraction of sdB stars may not be in binaries (Heber 2009; Fontaine et al. 2012). If true, this would require another formation channel, perhaps the singlestar evolution scenario proposed by Dorman et al. (1993). Their study of 105 single or wide-binary sdB stars showed that the binary evolution model of Han et al. (2002, 2003) overestimates the number of $\mathrm{sdB}$ stars formed through the white dwarf merger channel. In another scenario, the merger of a helium white dwarf with a low-mass hydrogen-burning star was proposed as a way of forming single sdB stars (Clausen \& Wade 2011). To distinguish between these evolutionary scenarios, orbital information on $\mathrm{sdB}$ star binaries is essential.

The existence of $\mathrm{sdB}$ pulsators ( $\mathrm{sdBV}$ ) was predicted by Charpinet et al. (1996). Independently, Kilkenny et al. (1997) discovered the first short-period sdBV star, EC 14026-2647. These stars are p-mode pulsators, where pulsations are driven by internal pressure fluctuations (Charpinet et al. 2000). The first long-period sdBV star, PG $1716+426$, is a g-mode pulsator (Green et al. 2003) in which gravity provides the restoring force. Some sdB stars have been discovered to exhibit both p-mode pulsations and g-mode pulsations. These objects are called hybrid pulsators (Oreiro et al. 2004; Schuh et al. 2006).

The pulsation periods of sdBV stars are usually stable (Østensen et al. 2001), and therefore they are good 
chronometers. A star's position in space may wobble due to the gravitational perturbations of a companion. From an observer's point of view, the light from the pulsating star is periodically delayed when it is on the far side of its orbit and advanced on the near side. Changes in the pulse arrival times are detected using the observed-minus-calculated $(\mathrm{O}-\mathrm{C})$ diagram. The $\mathrm{O}-\mathrm{C}$ diagram is a technique that has long been used in the binary star community to search for additional components, orbital period changes, mass loss, etc.

Several planets and substellar companions to sdB host stars have been detected by this method. Silvotti et al. (2007) were the first to detect a planet around the sdB star V391 Peg in this way. Lutz (2011) detected companions of the sdB stars HS 0444 +0458 and HS 0702+6043 that appear to be a brown dwarf and an exoplanet, respectively. Mullally et al. (2008) used the $O-C$ method to search for possible planets around DAV white dwarfs. Among the 15 white dwarf stars they surveyed, GD 66 exhibited $O-C$ variations consistent with a $2 M_{J}$ planet in a 4.5 year orbit. Also, several planets and companion stars to sdB host stars have been detected by the $O-C$ method using eclipse timings. A companion to the sdB star HS $0705+6700$ was similarly detected by Qian et al. (2009). While several authors have reported the existence of planets orbiting post-common envelope binaries, these conclusions must be regarded as tentative because they are based on data obtained over a time interval that is the same order as the orbital period proposed for an orbiting planet; further observations over several of the proposed orbital periods would be needed for confirmation. Applegate (1992) proposed an alternative explanation for eclipse timing variations, often referred to as the "Applegate mechanism," as a gravitational coupling of the orbit to changes in the shape of a magnetically active star in the system. Zorotovic \& Schreiber (2013) argued for additional observations to distinguish between the Applegate mechanism and planet formation following a common envelope phase. Planets may also survive a common envelope phase of their binary star host, as Kostov et al. (2016) and Veras et al. (2017) discuss. Völschow et al. (2016) concluded that an improved version of the Applegate mechanism, which includes angular momentum exchange between a finite shell and the stellar core, cannot uniquely explain orbital-period variations in the 16 systems they consider. A further possibility comes from calculations by Chen \& Podsiadlowski (2017) which indicate that observed orbital-period derivatives in two post-common envelope binaries involving a hot subdwarf (HW Vir and NY Vir) could be produced by a resonant interaction between the binary and a circumbinary disk having a mass in the range $10^{-4} M_{\odot}-10^{-2} M_{\odot}$.

EC 20117-4014 (=V4640 Sgr) is one of the first p-mode sdBV stars to be discovered, and it is known to be a spectroscopic binary system (O’Donoghue et al. 1997). This star was also selected by Otani (2015) as part of a three-year observational search for substellar companions among known sdB pulsators using the $O-C$ method. That project had two goals: (1) to determine whether the frequency of detectable companions supports the hypothesis that all, or nearly all, were formed via binary interactions and (2) to examine the frequency with which post main-sequence (MS) stars in general might harbor "planetary survivors." So far, we have a total of about 20 years of EC 20117-4014 photometric data. In this paper, we present the results of our $O-C$ analysis, and orbital information on the $\mathrm{sdB}$ and late F MS companion binary system.

Section 2 provides a summary of the physical properties of EC 20117-4014 and a discussion of the facilities and instrumentation used to obtain the data needed for the $O-C$ analysis. Section 3 outlines our reduction and analysis procedures. Section 4 presents our results derived from the observed pulsation peaks in the frequency spectrum of EC 20117-4014, and how they have been used to derive useful constraints on a previously reported F5 MS companion. Also, the star's evolutionary phase is discussed. Our conclusions and suggested additional work are summarized in Section 5.

\section{Target and Observations}

EC 20117-4014 (=V4640 Sgr) is an sdB star originally found in the Edinburgh-Cape (EC) Blue Object Survey (Stobie et al. 1997; Kilkenny et al. 2016). The composite spectra obtained by O'Donoghue et al. (1997) suggested that the sdB component of the EC 20117-4014 binary has a late F MS companion. This companion contributes more than half of the observed flux in the visual band. The apparent magnitude of the system is $V=12.47 \pm 0.01$, and the magnitude of the sdB star itself is $V=13.55 \pm 0.05$ (O'Donoghue et al. 1997). Three pulsation frequencies $(7.29 \mathrm{mHz} \quad(137.3 \mathrm{~s}), \quad 7.04 \mathrm{mHz} \quad(142.0 \mathrm{~s})$, and $6.35 \mathrm{mHz}(157.5 \mathrm{~s}))$ were detected by O'Donoghue et al. (1997), making EC 20117-4014 the fourth member of the class of short-period sdBV stars. Further high-speed photometry of EC 20117-4014 was obtained and an asteroseismology analysis was performed by Randall et al. (2006), who estimated the effective temperature $\left(32,800 \leqslant T_{\text {eff }} \leqslant 36,800 \mathrm{~K}\right)$, the gravity $(5.848 \leqslant$ $\log g \leqslant 5.864)$, and the mass $\left(0.50 \leqslant M_{\odot} \leqslant 0.59\right)$. According to Randall et al. (2006), the companion is confirmed to be a late $\mathrm{F}$ MS star, but they were unable to set strong constraints on its orbital period. Lynas-Gray (2013) found that the largest pulsation frequency $(7.29 \mathrm{mHz})$ exhibits day-to-day amplitude changes. Other sdBV stars, V541 Hya and KIC 010139564, show similar pulsation amplitude changes that are explained by rotational splitting (Randall et al. 2009; Baran et al. 2012). This suggests the pulsation amplitude changes of EC 20117-4014 may be a consequence of unresolved rotational splitting. The largest pulsation frequency amplitudes in the 2010-2011 data obtained by Otani (2015) showed a linear decrease with time.

The discovery observations of EC 20117-4014 were made with the 0.75 and $1.0 \mathrm{~m}$ telescopes at the Sutherland site of the South African Astronomical Observatory (SAAO) in 1995 (see Table 1 of O'Donoghue et al. 1997). At that time, both telescopes had (S-11) photomultiplier-based photometers (the response of the system has a similar effective wavelength to the Johnson B, but with a much broader bandpass), though these were replaced by the UCTCCD photometer (e.g., O'Donoghue et al. 1996) in the late 1990s. The UCTCCD could be used on either telescope, and our 0.75 and $1.0 \mathrm{~m}$ data post-1996 were obtained with the UCTCCD. Because EC 20117-4014 is relatively bright, it was mainly monitored with the SAAO $0.5 \mathrm{~m}$ telescope between the discovery in 1995 and 2000. The $0.5 \mathrm{~m}$ was permanently equipped with a (GaAs) photomultiplier-based photometer until it was recently decommissioned. For the $0.75 \mathrm{~m}$ SAAO telescope observations in 2001 and 2011, UCTCCD was used with a Johnson B filter. Since 2010, EC 20117-4014 has also been monitored using the SARA-CT $0.6 \mathrm{~m}$ telescope at the Cerro Tololo InterAmerican Observatory (CTIO) in Chile. ${ }^{9}$ Figure 1 provides a finder chart for the target and comparison

\footnotetext{
9 The SARA Observatory $0.6 \mathrm{~m}$ telescope at Cerro Tololo, Chile, is owned and operated by the Southeastern Association for Research in Astronomy (saraobservatory.org).
} 


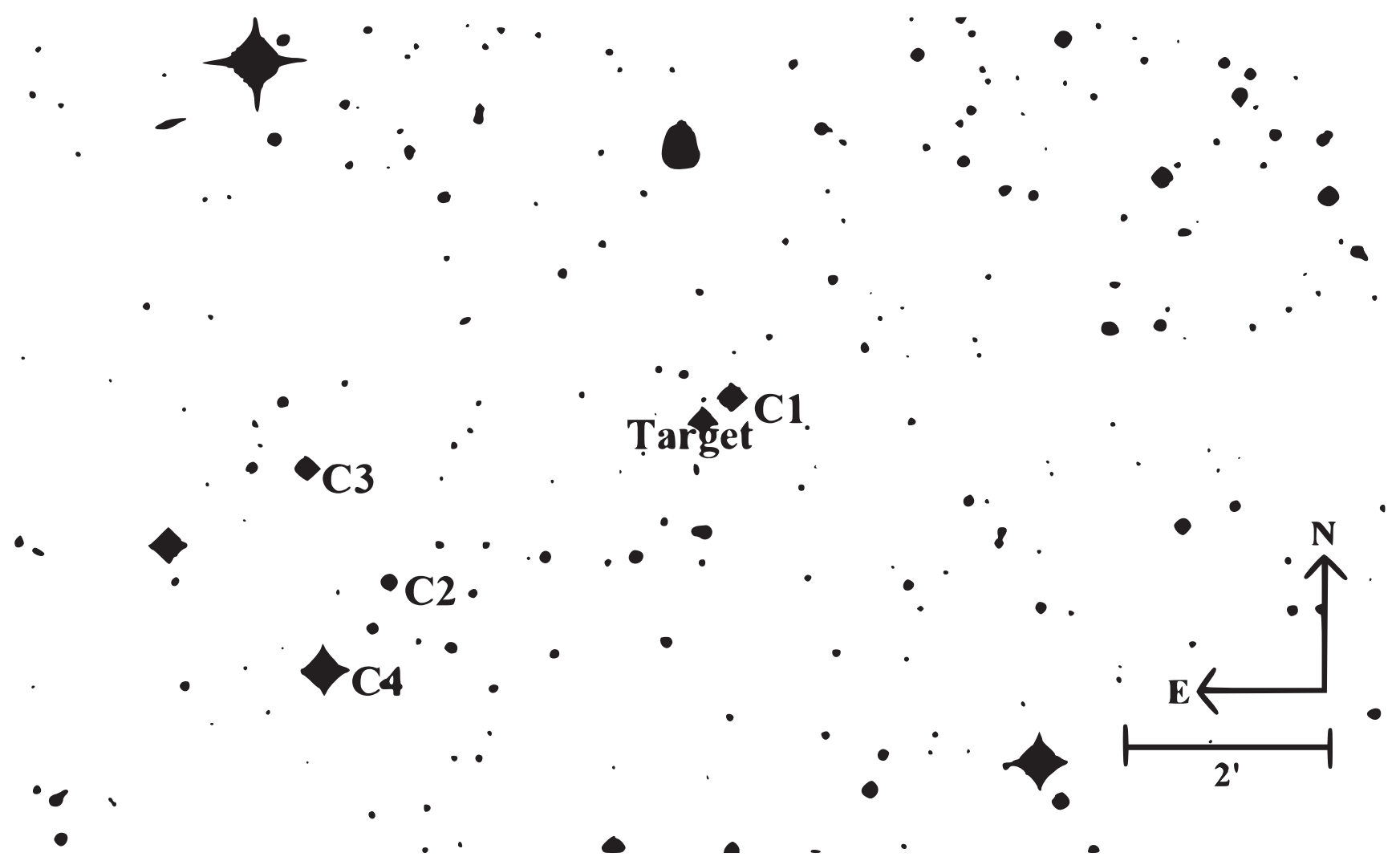

Figure 1. Finder Chart of EC 20117-4014 (http://simbad.u-strasbg.fr). The target and the comparison stars (C1-C4) are shown.

stars. For the SAAO CCD data, the only comparison star on the CCD chip was GSC 07952-01358 (C1 in Table 1). Therefore, this star was used as a comparison star for the SAAO CCD observations. For the SARA-CT observations, we used four comparison stars (including GSC 07952-01358). The coordinates of these target and comparison stars are given in Table 1. The observation log is presented in Table 2.

For the 0.6 m SARA-CT observations in 2010, 2011, and 2015, a Bessel-B filter was used. The Johnson-B and Bessel-B filters lie close enough in wavelength to have a negligible effect on the observed amplitudes of pulsation. The exposure time was $40 \mathrm{~s}$ for all runs in this study. For the data obtained in 2015, no filter was used, with an exposure time of $20 \mathrm{~s}$ because of a problem with the camera and filter wheel interface. To reduce read-out noise, $2 \times 2$ pre-binning was used for all images. The pulsations are not expected to be wavelength-independent (see Koen 1998). However, separate analyses were performed using only the data obtained in 1995-2011 and the cumulative data obtained in 1995-2015 to compare the results. The $O-C$ analysis results with and without the data obtained in 2015 were the same within the uncertainty level. Therefore, the result including the data observed in 2015 is presented in this paper.

\section{Data Reduction and $O-C$ method}

For the SARA-CT data, standard image-calibration procedures were performed using the Image Reduction and Analysis Facility (IRAF) ${ }^{10}$ to extract the raw intensity values for the target and comparison stars (Tody 1986, 1993). All flat fields

\footnotetext{
${ }^{10}$ IRAF is distributed by the National Optical Astronomy Observatories, which is operated by the Association of Universities for Research in Astronomy, Inc. (AURA) under cooperative agreement with the National Science Foundation.
}

Table 1

Coordinates of the Target and Comparison Stars (epoch 2000) (http://simbad. u-strasbg.fr)

\begin{tabular}{lcc}
\hline \hline Identification Name & R.A. & Decl. \\
\hline Target & $20^{\mathrm{h}} 15^{\mathrm{m}} 04^{\mathrm{s}} .79$ & $-40^{\circ} 05^{\prime} 44^{\prime \prime} \cdot 2$ \\
C1 & $20^{\mathrm{h}} 15^{\mathrm{m}} 03^{\mathrm{s}} .23$ & $-40^{\circ} 05^{\prime} 29^{\prime \prime} 1$ \\
C2 & $20^{\mathrm{h}} 15^{\mathrm{m}} 22^{\mathrm{s}} .20$ & $-40^{\circ} 07^{\prime} 25^{\prime \prime} 2$ \\
C3 & $20^{\mathrm{h}} 15^{\mathrm{m}} 26^{\mathrm{s}} .68$ & $-40^{\circ} 06^{\prime} 12^{\prime \prime} 6$ \\
C4 & $20^{\mathrm{h}} 15^{\mathrm{m}} 25^{\mathrm{s}} .91$ & $-40^{\circ} 08^{\prime} 22^{\prime \prime} 4$ \\
\hline
\end{tabular}

Note. For SAAO observations, only C1 (GSC 07952-01358) was used, as this is the only comparison star in the field.

were exposed on a twilight sky. For each night's data, the aperture that gave the best signal-to-noise ratio $(\mathrm{S} / \mathrm{N})$ was chosen and sky annuli were used to subtract the sky background. These values were then divided by similarly extracted intensity values of non-variable comparison stars listed in Table 1. The SAAO photomultiplier data were reduced by removing sky background as a cubic spline fitted to occasional sky measurements and then correcting for atmospheric extinction (see O'Donoghue et al. 1997 for more details). The SAAO CCD data were reduced using Dophot software (Schechter et al. 1993), which was modified by Darragh O'Donoghue (see Randall et al. 2006 and Lynas-Gray 2013 for details). For each night, the raw light curves were then normalized to the mean magnitude for that night. A secondorder polynomial was used to remove mild curvature in the light curves caused by differential extinction between the target and comparison stars. All times were corrected to Barycentric Julian Date (BJD). 
Table 2

Observation Log for EC 20117-4014

\begin{tabular}{|c|c|c|c|c|}
\hline Date & $\begin{array}{c}\text { Start of Run } \\
\text { (BJD-2445000) }\end{array}$ & $\begin{array}{c}\text { Length } \\
\text { (hr) }\end{array}$ & Observatory & Publications using the Data \\
\hline 1995 May 31 & 4869.49293 & 4.4 & SAAO & 1 \\
\hline 1995 Aug 17 & 4947.23604 & 8.0 & SAAO & 1 \\
\hline 1995 Aug 18 & 4948.23415 & 2.7 & SAAO & 1 \\
\hline 1995 Aug 25 & 4955.32857 & 4.1 & SAAO & 1 \\
\hline 1995 Aug 27 & 4957.25274 & 5.8 & SAAO & 1 \\
\hline 1995 Sep 19 & 4980.26625 & 4.3 & SAAO & 1 \\
\hline 1996 Mar 26 & 5168.58856 & 1.4 & SAAO & $\cdots$ \\
\hline 1996 Apr 21 & 5194.61163 & 1.0 & SAAO & $\ldots$ \\
\hline 1996 Apr 22 & 5195.60918 & 1.3 & SAAO & $\ldots$ \\
\hline 1996 May 26 & 5229.55177 & 1.5 & SAAO & $\ldots$ \\
\hline 1996 May 27 & 5230.55181 & 1.5 & SAAO & $\ldots$ \\
\hline 1996 Jun 28 & 5262.53746 & 1.0 & SAAO & $\cdots$ \\
\hline 1996 Jul 7 & 5272.48776 & 1.1 & SAAO & $\ldots$ \\
\hline 1996 Jul 11 & 5276.46623 & 1.1 & SAAO & $\ldots$ \\
\hline 1996 Jul 16 & 5281.47772 & 1.0 & SAAO & $\cdots$ \\
\hline 1996 Jul 20 & 5285.45608 & 1.0 & SAAO & $\cdots$ \\
\hline 1996 Aug 20 & 5316.44773 & 1.1 & SAAO & $\ldots$ \\
\hline 1996 Aug 21 & 5317.47060 & 1.1 & SAAO & $\cdots$ \\
\hline 1996 Sep 4 & 5331.41769 & 1.6 & SAAO & $\cdots$ \\
\hline 1996 Sep 6 & 5333.32821 & 1.1 & SAAO & $\ldots$ \\
\hline 1996 Sep 8 & 5335.31267 & 1.3 & SAAO & $\cdots$ \\
\hline 1996 Sep 9 & 5336.37706 & 1.5 & SAAO & $\cdots$ \\
\hline 1996 Sep 19 & 5346.36252 & 1.5 & SAAO & $\cdots$ \\
\hline 1996 Sep 20 & 5347.34214 & 1.5 & SAAO & $\ldots$ \\
\hline 1996 Sep 22 & 5349.34722 & 1.5 & SAAO & $\ldots$ \\
\hline 1996 Sep 23 & 5350.37251 & 1.4 & SAAO & $\ldots$ \\
\hline 1996 Oct 12 & 5369.28318 & 1.5 & SAAO & $\ldots$ \\
\hline 1996 Oct 13 & 5370.26306 & 1.5 & SAAO & $\cdots$ \\
\hline 1996 Oct 14 & 5371.27835 & 0.8 & SAAO & $\ldots$ \\
\hline 1996 Oct 27 & 5384.27263 & 1.5 & SAAO & $\cdots$ \\
\hline 1996 Oct 28 & 5385.26177 & 1.2 & SAAO & $\cdots$ \\
\hline 1997 Apr 30 & 5568.55880 & 2.6 & SAAO & $\cdots$ \\
\hline 1997 May 2 & 5570.57476 & 2.2 & SAAO & $\ldots$ \\
\hline 1997 May 4 & 5572.59402 & 2.0 & SAAO & $\ldots$ \\
\hline 1997 May 5 & 5573.62744 & 1.1 & SAAO & $\ldots$ \\
\hline 1997 May 9 & 5577.59845 & 1.9 & SAAO & $\cdots$ \\
\hline 1997 May 10 & 5578.63896 & 1.1 & SAAO & $\cdots$ \\
\hline 1998 Apr 24 & 5927.58573 & 1.8 & SAAO & $\cdots$ \\
\hline 1998 May 13 & 5946.57851 & 1.8 & SAAO & $\cdots$ \\
\hline 1998 May 31 & 5964.59448 & 1.9 & SAAO & $\cdots$ \\
\hline 1998 Jun 25 & 5989.57012 & 2.1 & SAAO & $\ldots$ \\
\hline 1998 Jul 18 & 6012.52173 & 2.1 & SAAO & $\ldots$ \\
\hline 1998 Jul 19 & 6014.39887 & 3.1 & SAAO & $\ldots$ \\
\hline 1998 Jul 21 & 6016.41143 & 2.0 & SAAO & $\ldots$ \\
\hline 1998 Aug 23 & 6049.32641 & 2.0 & SAAO & $\ldots$ \\
\hline 1998 Aug 24 & 6050.33752 & 2.6 & SAAO & $\ldots$ \\
\hline 1998 Sep 14 & 6071.31762 & 2.5 & SAAO & $\cdots$ \\
\hline 1998 Sep 17 & 6074.30708 & 2.2 & SAAO & $\ldots$ \\
\hline 1998 Sep 18 & 6075.29670 & 2.1 & SAAO & $\cdots$ \\
\hline 1998 Oct 6 & 6093.29647 & 2.2 & SAAO & $\ldots$ \\
\hline 1998 Oct 11 & 6098.30046 & 2.3 & SAAO & $\ldots$ \\
\hline 1999 May 24 & 6322.60764 & 1.6 & SAAO & $\ldots$ \\
\hline 1999 May 26 & 6324.57515 & 2.4 & SAAO & $\cdots$ \\
\hline 1999 Jul 7 & 6367.48358 & 2.7 & SAAO & $\ldots$ \\
\hline 1999 Jul 9 & 6368.51603 & 2.3 & SAAO & $\ldots$ \\
\hline 1999 Aug 8 & 6399.47168 & 2.2 & SAAO & $\cdots$ \\
\hline 1999 Aug 11 & 6402.41024 & 2.0 & SAAO & $\ldots$ \\
\hline 1999 Aug 13 & 6404.37116 & 2.1 & SAAO & $\ldots$ \\
\hline 1999 Aug 15 & 6406.47675 & 1.9 & SAAO & $\ldots$ \\
\hline 1999 Aug 16 & 6407.50784 & 1.1 & SAAO & $\ldots$ \\
\hline 1999 Sep 5 & 6427.27758 & 2.2 & SAAO & $\cdots$ \\
\hline 1999 Sep 17 & 6439.26778 & 2.2 & SAAO & $\ldots$ \\
\hline 1999 Oct 6 & 6458.26685 & 2.1 & SAAO & $\ldots$ \\
\hline
\end{tabular}


Table 2

(Continued)

\begin{tabular}{|c|c|c|c|c|}
\hline Date & $\begin{array}{l}\text { Start of Run } \\
\text { (BJD-2445000) }\end{array}$ & $\begin{array}{l}\text { Length } \\
(\mathrm{hr})\end{array}$ & Observatory & Publications using the Data \\
\hline 1999 Oct 13 & 6465.27847 & 2.2 & SAAO & $\cdots$ \\
\hline 2001 Jul 12 & 7103.31649 & 8.4 & SAAO & 2 \\
\hline 2001 Jul 13 & 7104.33424 & 7.9 & SAAO & 2 \\
\hline 2001 Jul 14 & 7105.30592 & 8.4 & SAAO & 2 \\
\hline 2010 Oct 14 & 10483.50415 & 4.2 & SARA-CT & 3 \\
\hline 2010 Oct 16 & 10485.55376 & 3.1 & SARA-CT & 3 \\
\hline 2011 Jun 11 & 10723.62516 & 4.0 & SARA-CT & 3 \\
\hline 2011 Jun 12 & 10724.64718 & 7.2 & SARA-CT & 3 \\
\hline 2011 Jul 20 & 10762.62790 & 4.5 & SARA-CT & 3 \\
\hline 2011 Jul 21 & 10763.53925 & 9.3 & SARA-CT & 3 \\
\hline 2011 Aug 3 & 10777.25923 & 4.3 & SAAO & 4 \\
\hline 2011 Aug 6 & 10780.26951 & 7.5 & SAAO & 4 \\
\hline 2011 Aug 7 & 10781.22805 & 8.5 & SAAO & 4 \\
\hline 2011 Aug 8 & 10782.20517 & 9.0 & SAAO & 4 \\
\hline 2011 Aug 9 & 10783.21753 & 8.6 & SAAO & 4 \\
\hline 2011 Aug 14 & 10788.22207 & 6.3 & SAAO & 4 \\
\hline 2011 Aug 15 & 10789.20804 & 8.4 & SAAO & 4 \\
\hline 2011 Aug 16 & 10790.20824 & 8.4 & SAAO & 4 \\
\hline 2011 Aug 20 & 10793.68243 & 3.8 & SARA-CT & 3 \\
\hline 2011 Aug 21 & 10794.52048 & 5.2 & SARA-CT & 3 \\
\hline 2011 Sep 19 & 10823.48896 & 5.8 & SARA-CT & 3 \\
\hline 2011 Sep 20 & 10824.52978 & 4.8 & SARA-CT & 3 \\
\hline 2011 Sep 21 & 10825.50645 & 5.3 & SARA-CT & 3 \\
\hline 2015 Jun 16 & 12189.69805 & 5.8 & SARA-CT & $\cdots$ \\
\hline 2015 Jun 17 & 12190.71299 & 4.2 & SARA-CT & $\ldots$ \\
\hline 2015 Jun 18 & 12191.69198 & 6.0 & SARA-CT & $\ldots$ \\
\hline
\end{tabular}

References (1) O’Donoghue et al. 1997; (2) Randall et al. 2006; (3) Otani 2015; (4) Lynas-Gray 2013.

The normalized light curves were then analyzed using Period04 (Lenz 2004). To improve detection and characterization of the pulsation frequencies and amplitudes, the data were pre-whitened. The pre-whitening technique was originally introduced by (Blackman \& Tukey 1958). After one or more frequencies are identified in the amplitude spectrum, they are removed from each light curve by subtracting the corresponding least-squares fitted sine curve (Sullivan et al. 2008). This analysis was performed for each of the runs listed in Table 2. The data exhibited three pulsation peaks that matched previously published data for EC 20117-4014. Only the two largest pulsations (F1 and F2) were $4 \sigma$ above the noise background, a threshold commonly used in the variable star community (see Breger et al. 1999). Therefore, only these two pulsations were used in the $\mathrm{O}-\mathrm{C}$ analysis.

In the $O-C$ method, the "observed" times of pulsation maxima are compared with "calculated" times of pulsation maxima, providing a sensitive way to detect period changes of astrophysical phenomena. A good review of the method can be found in Paparo et al. (1988).

The $O-C$ will be constant and flat if no period changes are occurring and the assumed period is correct. If the calculated period is constant but incorrect, the $O-C$ will be linear with a positive or negative slope. If the period is changing linearly with time (e.g., due to cooling or magnetic braking), the $O-C$ variations will exhibit a second-order polynomial of the form $c+b t+a t^{2}$, where $c=\Delta E_{0}, b=\Delta P E, a=\frac{1}{2} P \dot{P} E^{2}$, and $t$ is time. Here, $E$ is the integer number of cycles after the first observation; $P$ is the actual period of the pulsation; $\Delta E_{0}$ is the difference between observed and calculated reference epochs; $\Delta P$ is the difference between the actual period and the estimated period (see Winget \& Kepler 2008 for details); and $\dot{P}=d P / d t$. The precision of this technique, when applied to observations spanning several years, has allowed empirical measurement of the cooling rates of white dwarf stars and the evolution of sdB stars. So far, the $\dot{P} / P$ of sdB stars, V391 Peg, HS $0702+6043$, HS $0444+0458$ and CS 1246 are observed (Barlow et al. 2003; Silvotti et al. 2007; Lutz 2011).

If the $O-C$ diagram shows periodicities, it is most likely caused by the beating of two closely spaced pulsation frequencies or reflex motion caused by an unseen companion. The beating of two closely spaced frequencies, which may not be resolved in the power spectrum, causes not only sinusoidal variability in $\mathrm{O}-\mathrm{C}$ using pulsation timings, but also sinusoidal variability in pulsation amplitudes (Lutz 2011). In this case, the pulsation amplitude variability is $90^{\circ}$ out of phase with pulsation timing $O-C$ variability. Therefore, $O-C$ sinusoidal variations due to the beating of two closely spaced frequencies are easily distinguished from the $O-C$ periodic variations caused by a companion. Before searching for the sinusoidal signature of a companion in the $O-C$ diagram, a polynomial fit due to the effects of changes in pulsation period were removed. To obtain orbital solutions from $O-C$ diagrams, the $O-C$ and orbital elements relationship of Irwin $(1952,1959)$ were used. The Levenberg-Marquart (LM) algorithm (Press et al. 1992) was applied to evaluate the parameters of the ephemeris.

To determine "observed" and "calculated" maxima, the same method as Silvotti et al. (2007) in detecting the planet V391 Peg b was used. At first, all pulsation periods were determined by Fourier analysis using all data sets. Then, the expected pulsation maxima (="calculated" maxima) were estimated by least squares fitting of a constant period sine curves using all 
data sets. This equation for the largest pulsation mode $(7.29 \mathrm{mHz})$ is shown below:

$$
T=2445000.0006750+0.0015881 \times E .
$$

On the other hand, "observed" maxima were determined by least-squares fitting of sine curves with the same constant pulsating periods using data for each night. Here, all three detected pulsations were used for fitting the data simultaneously. As all pulsation periods are much shorter than one night, many pulsations occurred on any given night of observation. The time of the maximum of that fit closest to the midpoint of the night of observation was adopted as the "observed" time of maxima $(\mathrm{O})$ estimated for that night. Because all of a given night's data were used, this procedure maximized the precision to which a given night's phasing could be determined.

The inclination of the orbit with respect to the sky plane, $i$, is given by the following equation:

$$
a_{\mathrm{sdB}} \sin i=c T,
$$

where $T$ is the $O-C$ amplitude, $a_{\mathrm{sdB}}$ is the sdB star semimajor axis, and $c$ is the speed of light. Thus pulse arrival timing variations can be detected only for the pulsating sdB star, not the companion. The mass function for the system using $O-C$ amplitude, $T$, is described as

$$
f=\frac{\left(M_{\mathrm{F}} \sin i\right)^{3}}{\left(M_{\mathrm{sdB}}+M_{\mathrm{F}}\right)^{2}}=\frac{4 \pi^{2} c^{3} T^{3}}{G P^{2}},
$$

where $M_{\mathrm{F}}$ and $M_{\mathrm{sdB}}$ are the $\mathrm{F}$ and the sdB star masses; $P$ is the orbital period; and $G$ is the gravitational constant.

\section{Results and Discussion}

\subsection{Seasonal Pulsation Amplitude Variations}

An example light curve for the night of 2011 September 19 and an amplitude spectrum for the 2011 September 19-21 observing run are displayed in Figures 2 and 3. The highest and the lowest detectable frequencies for the data are $12.5 \mathrm{mHz}$ and $0.10 \mathrm{mHz}$, respectively. All peaks detected were between 1 and $10 \mathrm{mHz}$. Three pulsation peaks were found, which are listed in Table 3. To obtain the frequency of the second-largest pulsation, the largest pulsation was pre-whitened. To obtain the frequency of the third-largest pulsation, the largest and the second-largest pulsation were pre-whitened. These peaks matched the previously published results of O'Donoghue et al. (1997) and Randall et al. (2006) within the formal uncertainties. Peaks at $8.35 \mathrm{mHz}$ are distinguished in some of the 1995 and 2001 data, but the $\mathrm{S} / \mathrm{N}$ was less than $4 \sigma$ (this $\sigma$ is the average Fourier analysis spectrum amplitude after prewriting all three pulsations and this $8.35 \mathrm{mHz}$ signal) and the peak was not detected in other data, so this peak was not included in Table 3. This $8.35 \mathrm{mHz}$ peak is seen only in the data, which is obtained from the SAAO $0.75 \mathrm{~m}$ telescope. This frequency is known to be due to the telescope drive of the SAAO $0.75 \mathrm{~m}$ telescope in use at the time.

Because seasonal amplitude variations were detected in the largest pulsation frequency by Otani (2015), the possibility of seasonal amplitude changes was investigated. Seasonally binned pulsation amplitude variations as a function of date are shown in the top panel of Figure 4. These seasonal pulsation amplitude data points are weighted mean amplitudes of each year's data. This weight was obtained from $w=1 / \sigma_{i}^{2}$, where $\sigma_{i}$ is the pulsation amplitude uncertainty for each night. Triangles represent amplitudes of the largest amplitude pulsation frequency (F1: $7.29 \mathrm{mHz}$ ). Diamonds represent amplitudes of the second-largest amplitude pulsation frequency (F2: $7.04 \mathrm{mHz}$ ), and squares denote amplitudes of the thirdlargest amplitude pulsation frequency (F3: $6.35 \mathrm{mHz}$ ). The F2 mode was not detected in the data observed in 2015, and the F3 mode was not detected in the data observed in 2001 and 2015. These seasonal amplitude changes appear to be consistent for all three frequencies, although the F3 variations are comparable with the uncertainties and their reality is questionable. This figure shows that the amplitude of the F1 mode has been decreasing since 2010 at a rate of $0.0021 \mathrm{mmag}$ per day. The pulsation amplitude of the data observed in 2015 is almost onethird of the pulsation amplitude of the data observed in 2010. To test whether the changes in amplitudes are correlated, we compared the ratio of the seasonal amplitude of F1 to F2 and with F3. These results are shown in the bottom panel of Figure 4. Both ratios fit zero-slope straight lines within their uncertainties. The weighted average ratios are $0.38 \pm 0.03$ for $\mathrm{F} 2 / \mathrm{F} 1$, and $0.28 \pm 0.02$ for $\mathrm{F} 3 / \mathrm{F} 1$. While the seasonal amplitude variation is consistent in all pulsations, the F1 amplitude decline from 2010 to 2015 (in particular) cannot be understood to be a consequence of rotational splitting. Nonetheless, it is of interest to note that Hutchens et al. (2017, their Figure 1) observe a similar amplitude decline in the singlemode sdB pulsator CS 1246.

\subsection{O-C Periodic Variation due to the Existence of the Companion}

The first and second-largest amplitude pulsations F1 and F2 were used for an $\mathrm{O}-\mathrm{C}$ analysis. The $\mathrm{S} / \mathrm{N}$ of the third-largest pulsations (F3) for each night were less than $4 \sigma$, so this mode was not used in our analysis. Two distinct causes of period variation were identified in both $\mathrm{F} 1$ and $\mathrm{F} 2(\mathrm{O}-\mathrm{C})$ values. In addition to the near sinusoidal variation due to light-travel-time changes caused by the reflex motion of the pulsating sdB star, a second-order polynomial variation attributable to $\mathrm{sdB}$ star evolution was also discernible. Orbital and evolution contributions were fitted simultaneously; in fitting orbits, a single offset was established so as to minimize the difference between the F1 and F2 orbits. The pulsation period change is $\dot{P}=5.4$ $( \pm 0.7) \times 10^{-14} \mathrm{~d} \mathrm{~d}^{-1}$. The removed second-order polynomial curves for $\mathrm{O}-\mathrm{C}$ diagrams is

$$
\begin{aligned}
O-C= & \left(2.6( \pm 0.4) \times 10^{-6}\right) t^{2}-(4.0( \pm 0.6) \\
& \left.\times 10^{-2}\right) t+1.5( \pm 0.2) \times 10^{2}
\end{aligned}
$$

where $t$ is BJD-2445000.

The rate of period change $(\dot{P})$ indicates the age of the $\mathrm{sdB}$ star after the zero-age extreme horizontal branch (ZAEHB) (Charpinet et al. 2002). For p-modes, $\dot{P}$ is positive during the first evolutionary phase, which is before the thermonuclear fuel in its center is exhausted. $\dot{P}$ is negative during the second evolutionary phase, which is after the depletion of thermonuclear fuel in its center and before the post-EHB evolution. The change of sign occurs around 87-91 Myr after the ZAEHB. The positive values of $\dot{P}$ for EC 20117-4014 (sdB) denote that the the star is still in the first evolutionary phase. The age of EC 20117-4014 (sdB) can also be estimated from its effective temperature and surface gravity $g$. Figure 1 of Fontaine et al. (2012) indicates that the age of EC 20117-4014 

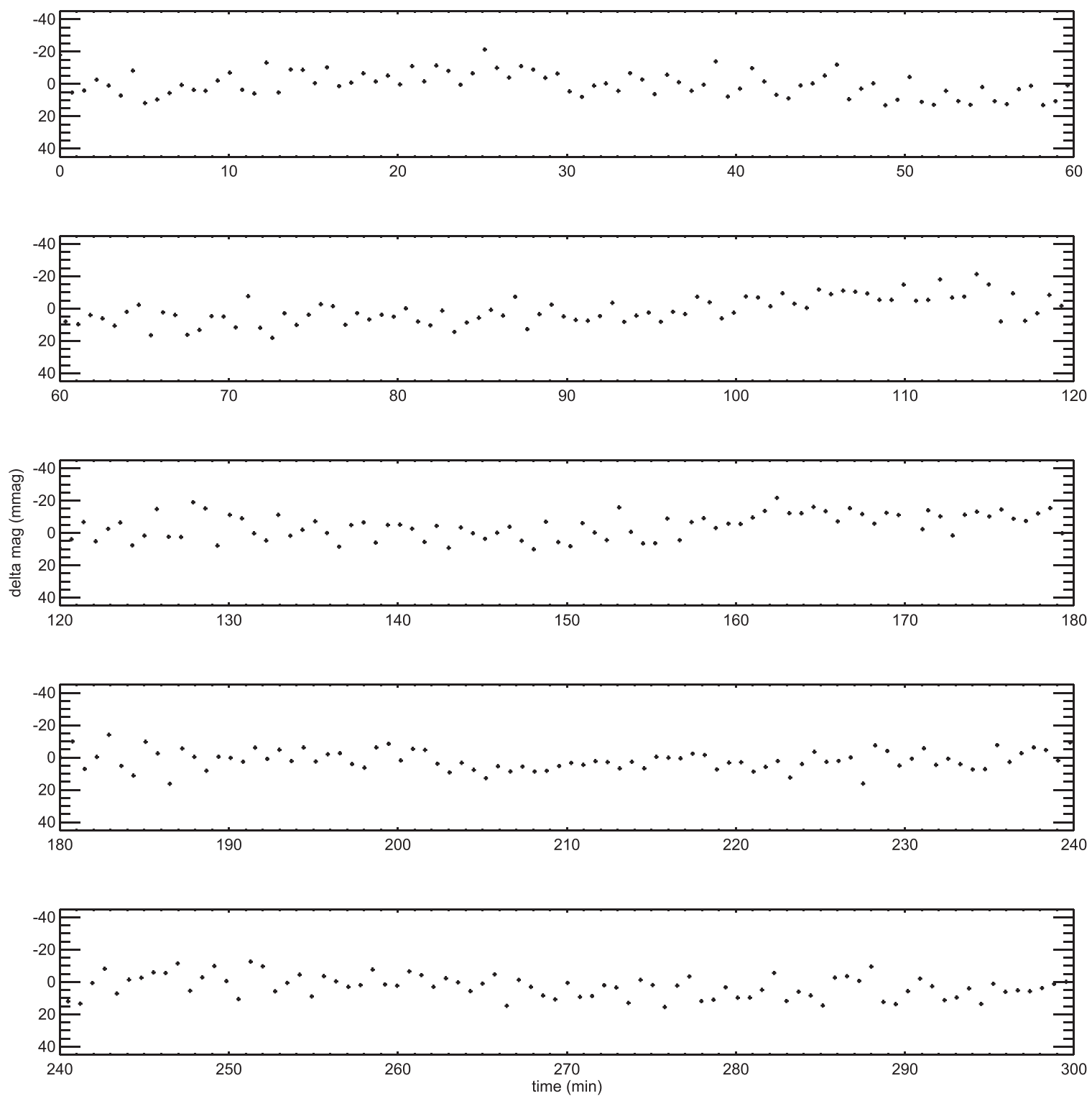

Figure 2. Example light curve for EC 20117-4014 (2011 September 19). All light curves are available in the figure set.

(The complete figure set (nine images) is available.)

(sdB) is $90 \pm 5 \mathrm{Myr}$. Therefore, the sdB component of $\mathrm{EC}$ 20117-4014 is about to end its core helium-burning phase.

Also, we can set an upper limit on the age of the sdB star since the zero-age MS. Choi et al. (2016) suggests that the MS lifetime of a $1.5 M_{\odot}$ star, like the late F MS companion, is about $2.5 \mathrm{Gyr}$ (see Figure 12 of their article). Assuming that both the sdB star and its F companion are coeval, an upper limit to the age of the sdB star is therefore $2.5 \mathrm{Gyr}$. However, this argument is not valid if large mass exchange from sdB progenitor to the companion occurred during the RLOF evolution.

The timescale for radius change is also obtained from the timescale for period change:

$$
\frac{\dot{P}}{P} \approx \frac{3}{2} \frac{\dot{R}}{R}
$$

where $R$ is the radius of the star. For EC 20117-4014, the timescale for period change $(P / \dot{P})$ is roughly $8.0 \times 10^{7}$ years. This value corresponds to a timescale for the radius increase of $R / \dot{R} \approx 1.2 \times 10^{8}$ years. According to Figure 3 of Kawaler (2010), $R / \dot{R} \approx 2 \times 10^{8}$ years at the first phase of core helium burning, and it will turn negative at the second phase of shell burning. $R / \dot{R}$ for the sdB component of EC 20117-4014 is about a half of $2 \times 10^{8}$ years, and this also indicates that this star's core burning is about to terminate.

Figure 5 presents the phase-folded $O-C$ diagrams for $\mathrm{F} 1$ and F2 after the removal of polynomials given by Equation (4). For F2, the only nights shown are those for which the pulsation amplitude is $4 \sigma$ above the noise level. The pulsation periods used are $137.2729 \mathrm{~s}$ for $\mathrm{F} 1$ and $142.1477 \mathrm{~s}$ for F2. Table 4 lists all $O-C$ data points for $\mathrm{F} 1$ and $\mathrm{F} 2$ pulsation modes, respectively. The solid curves in Figure 5 are the best-fitting 


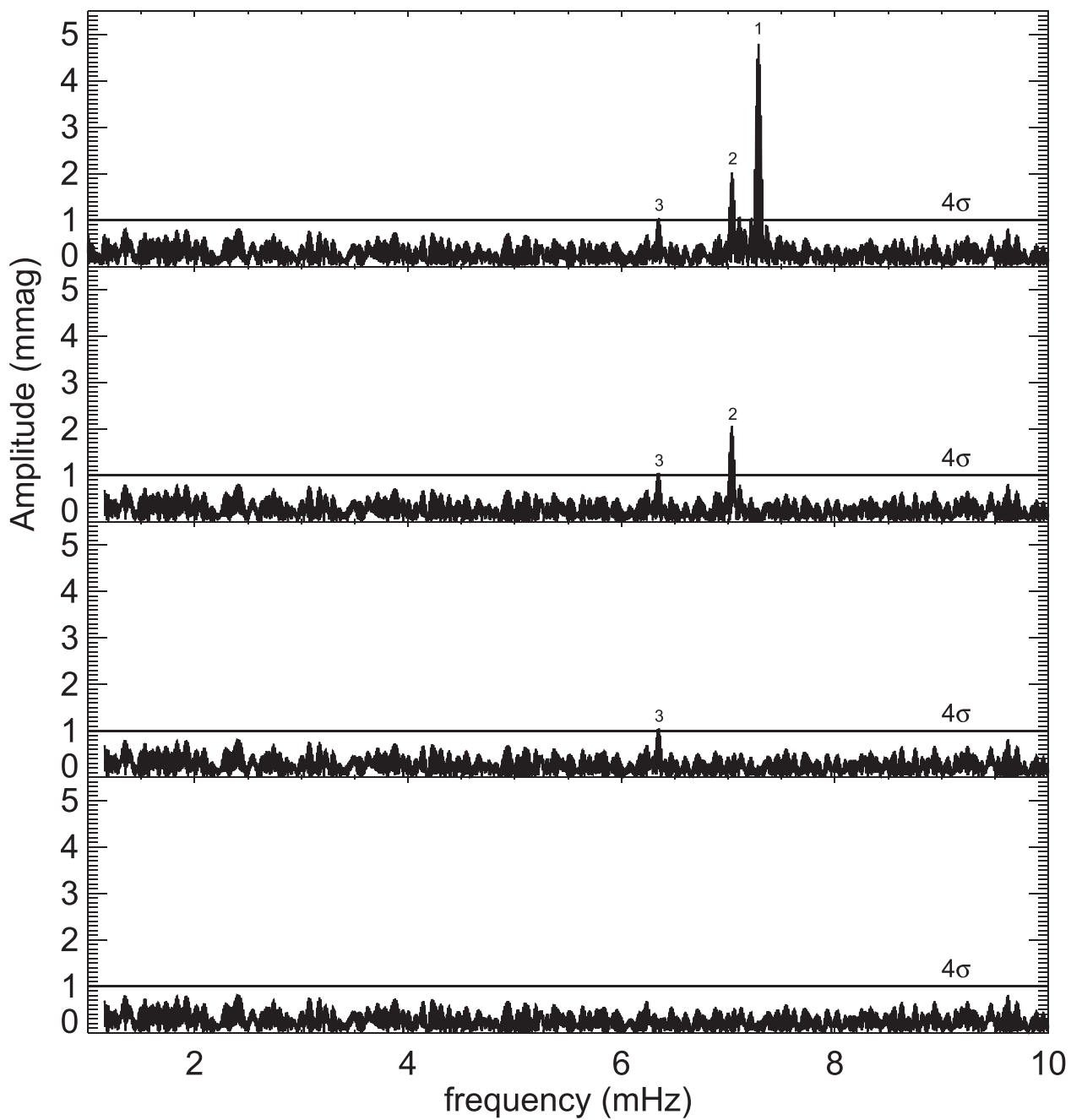

Figure 3. Example Fourier analysis for EC 20117-4014 (2011 September 19-21). The top panel shows the three most significant pulsation peaks (indicated by numbers) and the $4 \sigma$ threshold for significance adopted here. The lower panels show the successive steps of pre-whitening by sequentially removing the next largest pulsation peaks. The bottom panel shows the residual after all three pulsation peaks have been removed. In each panel, the horizontal lines indicate $4 \sigma$ noise levels. Fourier transform of each observing night data are available in the figure set.

(The complete figure set (nine images) is available.)

Table 3

Pulsation Peak Frequencies of EC 20117-4014

\begin{tabular}{lccccc}
\hline \hline Pulsation Mode & $\begin{array}{c}\text { Freq } \\
(\mathrm{mHz})\end{array}$ & $\begin{array}{c}\text { Freq } \sigma \\
(\mathrm{mHz})\end{array}$ & $\begin{array}{c}\text { Period } \\
(\mathrm{s})\end{array}$ & $\begin{array}{c}\text { Amplitude } \\
(\mathrm{mmag})\end{array}$ & $\begin{array}{c}\text { Amp } \sigma \\
(\mathrm{mmag})\end{array}$ \\
\hline F1 & 7.28484 & $1.10 \mathrm{E}-04$ & 137.2 & 4.3 & 0.2 \\
F2 & 7.03529 & $2.50 \mathrm{E}-04$ & 142.1 & 2.0 & 0.2 \\
F3 & 6.35145 & $5.30 \mathrm{E}-04$ & 157.4 & 0.9 & 0.2 \\
\hline
\end{tabular}

orbital solutions. The orbital periods are $792.3 \pm 0.3$ days, and these are the same within the uncertainties. Initially, we inadvertently omitted 1998 data from the $(O-C)$ diagram. When the 1998 data were included, these fitted the already established $O-C$ curve well. The best fitted orbital solutions for $\mathrm{F} 1$ and $\mathrm{F} 2 \mathrm{O}-\mathrm{C}$ data points are shown in Table 5. The formal $\chi^{2}$ values are $86.5(\mathrm{~F} 1)$ and $27.4(\mathrm{~F} 2)$, respectively. Degrees of freedom of F1 and F2 are 84 and 30. The corresponding right tail $p$-values are 0.40 and 0.60 . Therefore, the correlation between the periodicity of the $O-C$ data points and the binary motion with $P=792.3$ days are not rejected. The chi-squared values being consistent with the number of degrees of freedom suggests that all relevant physical information has been extracted from the data; in particular, there is no evidence for a third body associated with the EC 20117-4014 binary which would not have been accounted for in our model.

The resulting period of a periodic curve $(P=792.3$ days $)$ does not match sinusoidal variabilities in the F1 and F2 pulsation amplitudes, so these are not due to the beating of two closely spaced pulsation frequencies. Therefore, we concluded that the resulting variations are due to the light-travel effects caused by the F-type companion.

Most subdwarf-B (sdB) stars in binary systems have companions that are white dwarfs or M-dwarf MS stars (Kupfer et al. 2015); these have short orbital periods $(\lesssim 10$ days) and are understood (Han et al. 2002, 2003; Xiong et al. 2017) to be a consequence of evolution in a common envelope. A few sdB binaries have longer orbital periods with a F- or G-type giant or MS star; EC 20117-4014 was confirmed in the present paper to be an example and Aznar Cuadrado \& Jeffery (2002), Németh et al. (2012), and Vos et al. (2017) discovered some additional systems. SdB stars in wide binaries are formed (Han et al. 2002, 2003) as a consequence of a red 


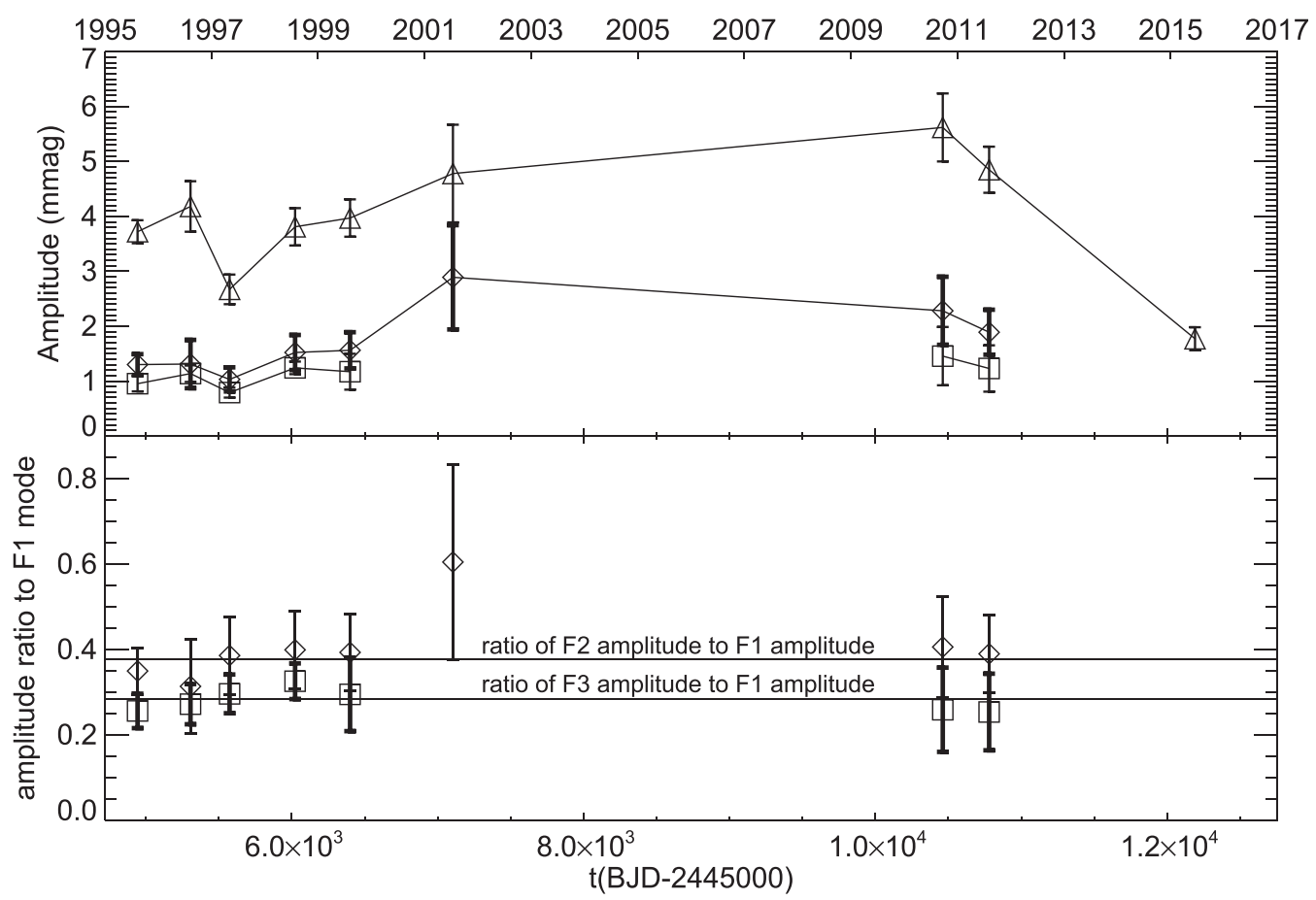

Figure 4. EC 20117-4014 seasonal pulsation amplitude variations of each pulsation mode (top) and pulsation amplitude ratios of the second and third-largest pulsation modes compared with the largest amplitude pulsation mode of $7.29 \mathrm{mHz}$ (bottom). Top: data points of the largest amplitude pulsation mode F1: $7.29 \mathrm{mHz}$ are marked by triangles. The points of the second and third-largest amplitude pulsation modes, F2 $(7.04 \mathrm{mHz})$ and F3 $(6.35 \mathrm{mHz})$, are marked by diamonds and squares, respectively. Each data point is the weighted average amplitude during each year (1995, 1996, 1997, 1999, 2001, 2010, 2011, and 2015). The F2 mode was not detected in the data observed in 2015, and the F3 mode was not detected in the data observed in 2001 and 2015. The F1 mode and F2 modes show obvious pulsation amplitude variations. Seasonal amplitude variations appear to be consistent for all three frequencies, although F3 mode amplitude variations are too small (compared to the uncertainty) to confirm the variations are real. Bottom: F2 mode (diamonds) and F3 mode (squares) pulsation amplitudes compared to F1 amplitudes. Both ratios fit straight lines. The horizontal lines are the weighted average ratios of both frequencies, which is $0.38 \pm 0.03$ for amplitude ratios F2/F1, and $0.28 \pm 0.02$ for F3/F2.

giant progenitor losing almost all of its hydrogen envelope, at the onset of core helium burning, through stable Roche lobe overflow (RLOF); their calculations suggest that the orbits should be circular and have periods $\lesssim 500$ days.

Orbital element determination for long-period $\mathrm{sdB}$ binaries presents a greater challenge than for those with short periods. Radial velocity observations by Østensen \& Van Winckel (2012), Deca et al. (2012), Barlow et al. (2012), Wade et al. (2014), identified sdB stars having a MS or giant companion with orbital periods $>500$ days, significantly greater than the Han et al. $(2002,2003)$ orbital-period distribution would suggest. Chen et al. (2013) reproduce the orbital-period distribution Østensen \& Van Winckel (2012) observe using detailed binary evolution calculations for the stable RLOF channel, improving on the simplified binary population synthesis by Han et al. (2003). The estimated period of binaries that went through this RLOF channel is $P=400-1100$ days. The derived orbital period of the EC 20117-4014 binary system $(P=792.3$ days $)$ falls in the middle of this range.

Figure 6 shows the residuals of the fitted orbital solution of $O-C$ data points shown in Figure 5. Vos et al. (2015, their Figure 1) plot orbital eccentricities against periods for those long-period sdB binaries for which orbital elements had been determined at the time of publication. A clear correlation is apparent with longer period systems having higher orbital eccentricities. Modifications to the binary module of the stellar evolution code Modules for Experiments in Stellar Astrophysics (Paxton et al. 2011, 2013, 2015, MESA) by Vos et al. (2015), to include eccentricity pumping processes, results in binary systems with observed orbital eccentricities when eccentricity pumping via a circumbinary disk is accompanied by phase-dependent RLOF. A remaining difficulty is the model prediction of some high orbital eccentricities for short periods which are at variance with available observations.

The deduced period of 792.3 days suggested an orbital eccentricity of $0-0.07$ on the basis of the Vos et al. (2015) figure 1. No significant eccentricity is detected in the EC 20117-4014 binary system, and the upper limit to the eccentricity is 0.025 if $3 \sigma$ is used as an upper limit. The eccentricity of this binary system has the lowest upper limit among all wide sdB binaries with known orbital parameters (Vos et al. 2017).

Using the periodicity shown in Figure 5 (792.3 \pm 0.3 days), which is due to the orbital motion, the semimajor axis for the star's orbit was calculated. If both the sdB star mass $\left(m_{\mathrm{sdB}}\right)$ and F star mass $\left(m_{\mathrm{F}}\right)$ are known, semimajor axes of both sdB and F MS stars are calculated using Equations (2) and (3). According to Randall et al. (2006), the EC 20117-4014 sdB star mass range is $m_{\mathrm{sdB}}=0.50-0.59 M_{\odot}$. However, the mass of the F star in the EC 20117-4014 binary is unknown. Therefore, the F stars' mass was estimated from the stellar type. As the estimated stellar type of this companion is F5V or late F MS (O'Donoghue et al. 1997), the MS mass range (SchmidtKaler 1982) of F5-G0 (which is $1.40-1.05 M_{\odot}$ ) was used to estimate the semimajor axis. The semimajor axes ranges of the sdB star and the F MS star are $a_{\mathrm{sdB}}=1.3-1.5 \mathrm{au}$ and $a_{\mathrm{F}}=0.5-0.7 \mathrm{au}$. The inclination was computed using possible mass ranges. The inclination range obtained is $i=37^{\circ} .9-48^{\circ} .0$. It is not surprising that eclipses have not been detected in the 


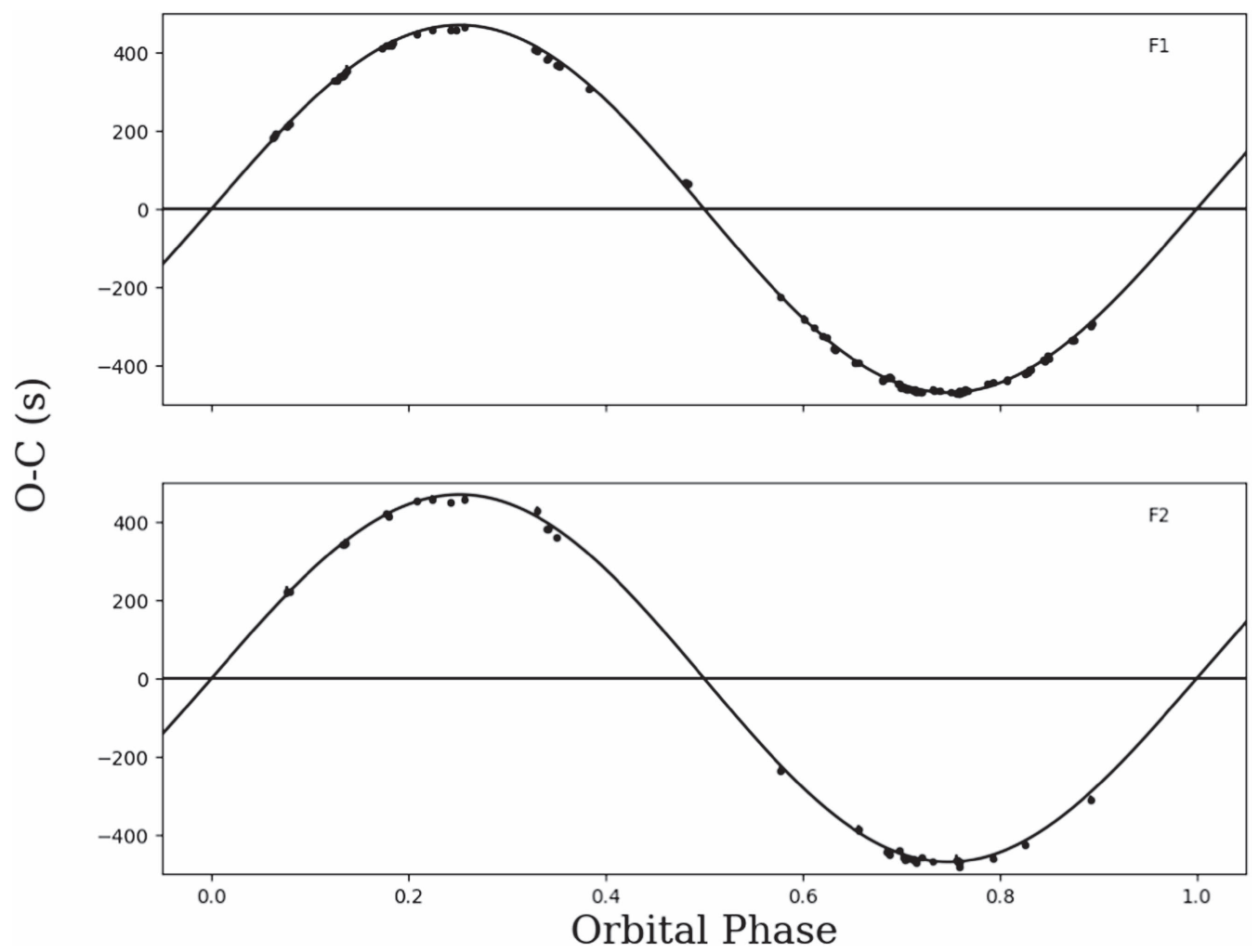

Figure 5. Phase-folded $O-C$ curves for EC 20117-4014 constructed (see the text) from the largest and the second-largest amplitude pulsations (F1: $7.29 \mathrm{mHz}$ at the top and F2: $7.04 \mathrm{mHz}$ at the bottom). The third-largest amplitude pulsation of each night was lower than $4 \sigma$ above the noise level, so it was not used for the $O-C$ analysis. Each square represents the $O-C$ value of each entire night. $O-C$ uncertainties of some data points are smaller than the symbol size. Both fitted curves have a period of 792.3 days and an amplitude of $468.9 \mathrm{~s}$.

observed light curves. The orbital parameters are shown in Table 5 .

In principle, the F MS companion should be detectable via radial velocity variations. However, radial velocities are difficult to measure for sdB stars because of their high gravity (which broadens the line profiles). Generally, the (nonrelativistic) radial velocity of the $\mathrm{sdB}$ star can be estimated using the mass function

$$
f=\frac{\left(M_{\mathrm{F}} \sin i\right)^{3}}{\left(M_{\mathrm{sdB}}+M_{\mathrm{F}}\right)^{2}}=\frac{P K^{3}}{2 \pi G},
$$

where $M_{\mathrm{sdB}}$ and $M_{\mathrm{F}}$ are the masses of the sdB star and $\mathrm{F}$ companion. $P, K$, and $G$ are the orbital period, radial velocity semi-amplitude, and gravitational constant, respectively. Comparing this mass function with the previously obtained mass function using $O-C$ amplitude, $T$, (see Equation (2)), the relationship between $K$ and $T$ is described as

$$
K=\frac{2 T c \pi}{P}
$$

This equation only depends on $T$ and $P$, and is not dependent on $M_{\mathrm{sdB}}$ and $M_{\mathrm{F}}$. Using this equation yields an estimate for the sdB star radial velocity amplitude of $12.90 \pm 0.03 \mathrm{~km} \mathrm{~s}^{-1}$.

Silvotti et al. (2014) succeeded in measuring the radial velocities of $\mathrm{sdB}$ stars to a precision of $50-100 \mathrm{~m} \mathrm{~s}^{-1}$ ( $5 \sigma$ level) using the $3.6 \mathrm{~m}$ Telescopio Nazionale Galileo. The EC 20117$4014 \mathrm{sdB}$ star radial velocity amplitude obtained is much larger than this, so it is possible to confirm the companion using the radial velocity method. However, as shown here, much smaller telescopes ( $1 \mathrm{~m}$ class) are adequate to detect it using the $O-C$ method.

\section{Conclusions}

This paper presents an orbital solution for the EC 201174014 binary and the evolutionary stage of its sdB component. The formation theory for sdB stars is still hotly debated and the frequency with which companions are found may help settle this issue. In particular, orbital element determination for longperiod $\mathrm{sdB}+\mathrm{F} / \mathrm{G}$ dwarf binaries is not as common as those with short periods, and only 12 binaries' orbital solutions were obtained so far. Among those systems, EC 20117 - 4014 is unique in that the orbital elements were obtained using the changing light-travel-time across the sdB star orbit, not using radial velocities. Photometric data of EC 20117-4014 for 20 years shows obvious periodic variations in both the largest and the second-largest amplitude pulsation frequencies and allowed an orbital solution for the F MS companion and the sdB star. The data indicate the orbital parameters of the binary system are

1. the orbital period $P=792.3 \pm 0.2$ days;

2. the upper limit of the eccentricity is 0.025 . This is the lowest upper limit of the eccentricity among all 12 wide sdB binaries with known orbital parameters;

3 . the light-travel-time amplitude $A=468.9 \pm 1.1 \mathrm{~s}$; and

4. the inclination $i=37^{\circ} .9-48^{\circ} .0$, which does not permit eclipses.

Importantly, the EC 20117-4014 sdB star represents another example of a system that has very likely experienced binary 
Table 4

$O-C$ Data Points for F1 $(7.29 \mathrm{mHz})$ and F2 $(7.04 \mathrm{mHz})$ Pulsation Modes before Removing the Second-order Polynomial Fit

\begin{tabular}{|c|c|c|c|c|c|c|c|c|c|}
\hline $\begin{array}{l}\text { Time } \\
\text { (BJD-2445000) }\end{array}$ & $\begin{array}{c}{[\mathrm{F} 1] \underset{O}{O}-\mathrm{C}} \\
\text { (s) }\end{array}$ & $\begin{array}{c}{[\mathrm{F} 1] O-C} \\
\text { Uncertainties } \\
(\mathrm{s})\end{array}$ & {$[\mathrm{F} 2] O-C$} & $\begin{array}{c}{[\mathrm{F} 2] O-C} \\
\text { Uncertainties }\end{array}$ & $\begin{array}{l}\text { Time } \\
\text { (BJD-2445000) }\end{array}$ & $\begin{array}{l}{[\mathrm{F} 1] \mathrm{O}-\mathrm{C}} \\
\text { (s) }\end{array}$ & $\begin{array}{c}{[\mathrm{F} 1] O-C} \\
\text { Uncertainties } \\
(\mathrm{s})\end{array}$ & $\begin{array}{c}{[\mathrm{F} 2] O-C} \\
(\mathrm{~s})\end{array}$ & $\begin{array}{c}\text { [F2] } O-C \\
\text { Uncertainties } \\
\text { (s) }\end{array}$ \\
\hline & & & & & 6427.272280 & 443.8 & 5.0 & 450.8 & 9.9 \\
\hline 4869.492986 & 470.0 & 2.0 & 472.0 & 5.5 & 6439.263252 & 453.4 & 3.8 & 456.6 & 10.8 \\
\hline 4947.402781 & 396.7 & 1.9 & 402.7 & 4.9 & 6458.263947 & 454.5 & 4.1 & $\ldots$ & $\ldots$ \\
\hline 4948.291201 & 397.5 & 2.9 & 402.0 & 8.9 & 6465.276215 & 459.9 & 3.2 & 456.4 & 10.6 \\
\hline 4955.328631 & 381.2 & 2.7 & 381.8 & 6.7 & 7103.488750 & 173.9 & 9.4 & $\ldots$ & $\ldots$ \\
\hline 4957.252794 & 376.9 & 2.3 & $\cdots$ & $\cdots$ & 7104.488620 & 177.6 & 6.1 & $\ldots$ & $\ldots$ \\
\hline 4980.266303 & 319.2 & 4.6 & $\cdots$ & $\cdots$ & 7105.474873 & 186.2 & $\begin{array}{l}0.1 \\
6.3\end{array}$ & $\ldots$ & $\ldots$ \\
\hline 5168.588619 & -315.1 & 3.6 & $\cdots$ & $\cdots$ & 10483.589470 & 417.7 & 5.7 & $\ldots$ & $\ldots$ \\
\hline 5194.611691 & -383.6 & 5.6 & $\cdots$ & $\cdots$ & 10485.617570 & 415.3 & 6.0 & 423.4 & 13.2 \\
\hline 5195.609237 & -381.7 & 4.6 & $\cdots$ & $\cdots$ & 10723.804710 & $\begin{array}{r}415.3 \\
-342.7\end{array}$ & $\begin{array}{l}0.0 \\
7.0\end{array}$ & $\begin{array}{l}423.4 \\
\ldots\end{array}$ & $\begin{array}{c}15.2 \\
\ldots\end{array}$ \\
\hline 5229.582828 & -438.1 & 5.2 & $\cdots$ & $\cdots$ & 10724.797720 & $\begin{array}{l}-342.1 \\
-347.6\end{array}$ & $\begin{array}{l}1.0 \\
4.8\end{array}$ & $\begin{array}{l}\cdots \\
\cdots\end{array}$ & $\begin{array}{l}\cdots \\
\cdots\end{array}$ \\
\hline 5230.582731 & -435.7 & 4.5 & -426.2 & 10.6 & 10762.721360 & $\begin{array}{l}-541.0 \\
-420.4\end{array}$ & $\begin{array}{l}4.0 \\
7.6\end{array}$ & $\cdots$ & $\cdots$ \\
\hline 5262.537515 & -457.3 & 7.0 & $\cdots$ & $\cdots$ & 10763.733280 & -423.9 & 4.6 & $\ldots$ & $\ldots$ \\
\hline 5272.487817 & -460.3 & 6.9 & $\cdots$ & $\cdots$ & 10777.350960 & -441.2 & 3.7 & $\ldots$ & $\ldots$ \\
\hline 5276.466287 & -462.0 & 5.6 & -451.1 & 18.5 & 10780.428190 & -443.8 & 2.5 & -440.3 & 5.4 \\
\hline 5281.489239 & -457.9 & 5.5 & $\cdots$ & $\cdots$ & 10781.391280 & -444.0 & 4.1 & -450.6 & 8.4 \\
\hline 5285.456137 & -454.3 & 4.8 & $\cdots$ & $\cdots$ & 10782.395440 & -445.4 & 3.0 & -444.7 & 8.5 \\
\hline 5316.471197 & -432.4 & 5.0 & $\cdots$ & $\cdots$ & 10783.396050 & -444.4 & 2.9 & -445.6 & 9.0 \\
\hline 5317.494223 & -427.2 & 6.3 & $\cdots$ & $\cdots$ & 10788.339390 & -448.9 & 3.3 & -448.5 & 16.1 \\
\hline 5331.450016 & -412.3 & 5.6 & -415.5 & 14.9 & 10789.383570 & -447.5 & 3.0 & -447.5 & 8.2 \\
\hline 5333.350491 & -409.7 & 8.1 & $\cdots$ & $\cdots$ & 10790.383670 & -451.5 & 2.3 & -455.8 & 6.6 \\
\hline 5335.340110 & -404.4 & 4.5 & $\cdots$ & $\cdots$ & 10793.761090 & -451.5 & 5.6 & $\ldots$ & $\cdots$ \\
\hline 5336.408112 & -402.8 & 7.3 & $\cdots$ & $\cdots$ & 10794.629430 & -452.3 & 3.6 & -442.2 & 8.8 \\
\hline 5346.393672 & -378.5 & 5.7 & $\cdots$ & $\cdots$ & 10823.610400 & -454.1 & 4.0 & -450.6 & 8.2 \\
\hline 5347.373323 & -383.0 & 5.8 & $\cdots$ & $\cdots$ & 10824.618790 & -454.9 & 4.4 & -454.2 & 9.2 \\
\hline 5349.378340 & -366.6 & 7.1 & $\cdots$ & $\cdots$ & 10825.615890 & -456.4 & 3.6 & -464.2 & 11.4 \\
\hline 5350.400659 & -375.8 & 7.6 & $\cdots$ & $\cdots$ & 12189.819940 & 108.8 & 4.8 & $\ldots$ & $\ldots$ \\
\hline 5369.315228 & -329.4 & 4.4 & $\cdots$ & $\cdots$ & 12190.800550 & 106.1 & 5.8 & $\ldots$ & $\ldots$ \\
\hline 5370.294472 & -326.8 & 6.3 & $\cdots$ & $\cdots$ & 12191.817640 & 104.6 & 5.4 & $\ldots$ & $\ldots$ \\
\hline 5371.294833 & -330.3 & 5.4 & $\ldots$ & $\ldots$ & & 104.0 & 0.4 & . & . \\
\hline
\end{tabular}

$5384.303378 \quad-291.9$

$5385.286270-287.2$

$5568.558797 \quad 332.6$

$5570.574757 \quad 334.5$

$5572.594017 \quad 343.5$

$5573.627437 \quad 342.5$

$5576.640367 \quad 354.5$

$5577.598447 \quad 354.2$

$5578.638957 \quad 358.3$

$5927.623752-223.2$

5946.615308

5954.636558

5964.633991

5989.612737

6012.564885

6014.466184

6016.453894

6049.365569

6050.390923

6071.370080

6074.351007

6075.339904

6093.341990

6098.348228

6322.602836

6324.570313

6367.457465

6368.507951

6399.464757

6402.403646

6404.364641

6406.470197

6407.501447
$-282.1$

$-302.7$

$-326.6$

$-391.4$

$-430.5$

$-429.7$

$-433.5$

$-462.3$

$-464.1$

$-464.4$

$-466.9$

$-462.8$

$-447.1$

$-444.6$

206.6

214.2

337.4

341.1

407.8

415.7

415.0

413.9

420.4

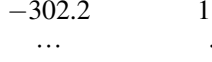

...

$\ldots$

$\cdots$

$\cdots$

$\cdots$

…

351.8

$-233.5$

...

$\cdots$

$-385.8$

$-443.5$

$-449.9$

...

$-466.5$

-..

$-466.8$

$\cdots$

$\cdots$

$-459.3$

219.0

218.9

342.1

340.2

‥

420.2

412.1

...
Table 4

(Continued)

Note. Time is mid-observing time.

(This table is available in its entirety in machine-readable form.)

Table 5

Orbital Information of the EC 20117-4014 System

\begin{tabular}{lc}
\hline \hline Parameters & Values \\
\hline Period (days) & $792.3 \pm 0.3$ \\
Amplitude (s) & $468.9 \pm 1.1$ \\
Eccentricity & $0.004 \pm_{0.004}^{0.007}$ \\
Zero point of time (BJD-2445000) & $4673.0 \pm 0.4$ \\
Radial velocity for sdB star $K_{\mathrm{sdB}}\left(\mathrm{km} \mathrm{s}^{-1}\right)$ & $12.90 \pm 0.03$ \\
Semimajor axis $a_{\mathrm{sdB}}(\mathrm{au})$ & $1.3-1.5$ \\
Semimajor axis $a_{\mathrm{F} 5}(\mathrm{au})$ & $0.5-0.7$ \\
Inclination (degrees) & $37.9-48.0$ \\
\hline
\end{tabular}

Note. Semimajor axes $a_{\mathrm{F}}$ and $a_{\mathrm{sdB}}$ and inclination $i$ are estimated ranges using possible sdB and $\mathrm{F}$ main-sequence star masses.

evolution, and lost almost all of its hydrogen envelope through stable RLOF. The 20 years of photometric data also showed the timescale for period changes $\dot{P} / P$. The $\dot{P} / P$ and the corresponding timescale for radius increase indicates that the $\mathrm{sdB}$ in EC 20117-4014 is about to deplete thermonuclear fuel in its center.

T.D.O. gratefully acknowledges support from National Science Foundation grants AST-108845, AST-1358787, and PHY-1358879. 


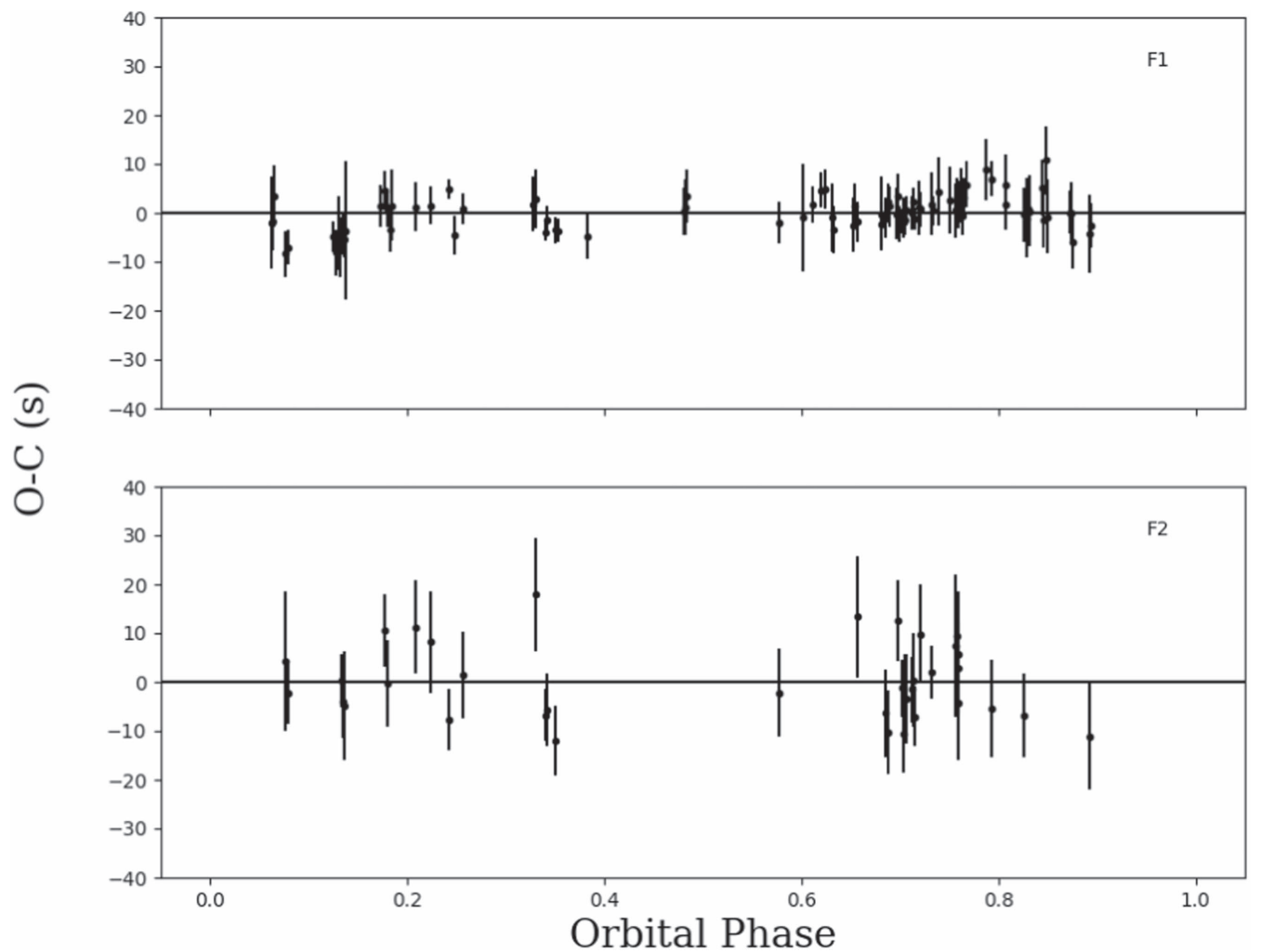

Figure 6. Residuals of the fitting of $O-C$ data points shown in Figure 5. The top panel shows the residual for F1 $O-C$ data points and the bottom panel shows the residual for F2 $O-C$ data points.

A.E.L.G. is indebted to the panel for the Allocation of Telescope Time (United Kingdom) for the award of travel and subsistence expenses.

This paper uses observations made at the South African Astronomical Observatory and we grateful for generous allocations of telescope time by the SAAO and for the continuing support at the telescope provided by SAAO staff. This paper is based partly upon work supported financially by the National Research Foundation of South Africa.

We are indebted to the anonymous referee for comments and suggestions which have improved our paper.

We are also indebted to Dr. J. Vos for providing us with a copy of his elliptical orbit fitting program.

Facilities: $\quad$ SAAO:0.5m, SAAO:0.75m, SAAO:1.0m, CTIO:0.6m.

Software: Period04 (Lenz 2004).

\section{ORCID iDs}

T. Otani 나 https://orcid.org/0000-0002-1691-8479

T. D. Oswalt (ㅁ) https://orcid.org/0000-0002-8541-9921

C. Koen (10) https://orcid.org/0000-0003-2291-2660

\section{References}

Applegate, J. H. 1992, ApJ, 385, 621

Aznar Cuadrado, R., \& Jeffery, C. S. 2002, A\&A, 385, 131

Baran, A. S., Reed, M. D., Stello, D., et al. 2012, MNRAS, 424, 2686

Barlow, B. N., Dunlap, B. H., Clemens, J. C., et al. 2003, A\&A, 398, 283

Barlow, B. N., Liss, S. E., Wade, R. A., \& Green, E. M. 2012, ApJ, 771, 23

Bergeron, P., Wesemael, F., Beauchamp, A., et al. 1994, ApJ, 432, 305

Blackman, R. B., \& Tukey, J. W. 1958, Bell Syst. Tech. J, 37, 185

Bonanno, A., Catalano, S., Frasca, A., Mignemi, G., \& Paternò, L. 2003, A\&A, 398,283
Breger, M., Handler, G., Garrido, R., et al. 1999, A\&A, 349, 225

Charpinet, S., Fontaine, G., Brassard, P., \& Dorman, B. 2000, ApJS, 131, 223

Charpinet, S., Fontaint, G., Brassard, P., \& Dorman, B. 1996, ApJL, 471, L103

Charpinet, S., Fontaint, G., Brassard, P., \& Dorman, B. 2002, ApJS, 140, 469

Chen, W.-C., \& Podsiadlowski, Ph. 2017, ApJL, 837, L19

Chen, X., Han, Z., Deca, J., \& Podsiadlowski, P. 2013, MNRAS, 434, 186

Choi, J., Dotter, A., Conroy, C., et al. 2016, ApJ, 823, 2

Clausen, D., \& Wade, R. A. 2011, ApJ, 733, L42

Deca, J., Marsh, T. R., Østensen, R. H., et al. 2012, MNRAS, 421, 2798

Dorman, B., Rood, R. T., \& O'Connell, R. W. 1993, ApJ, 419, 596

Efron, B. 1979, AnSta, 7, 1

Fontaine, G., Brassard, P., Charpinet, S., et al. 2012, A\&A, 539, 12

Green, E. M., Fontaine, G., Reed, M. D., et al. 2003, ApJ, 583, 31

Han, Z., Podsiadlowski, Ph., Maxted, P. F. L., \& Marsh, T. R. 2003, MNRAS, 341,669

Han, Z., Podsiadlowski, Ph., Maxted, P. F. L., Marsh, T. R., \& Ivanova, N. 2002, MNRAS, 336, 449

Heber, U. 2009, ARA\&A, 47, 211

Hutchens, Z. L., Barlow, B. N., Vasquez, S. A., et al. 2017, OAst, 26, 252

Irwin, J. B. 1952, ApJ, 116, 211

Irwin, J. B. 1959, AJ, 64, 149

Kawaler, S. D. 2010, AN, 331, 1020

Kilkenny, D., O'Donoghue, D., Koen, C., Stobie, R. S., \& Chen, A. 1997, MNRAS, 287, 867

Kilkenny, D., Worters, H. L., O’Donoghue, D., et al. 2016, MNRAS, 459,4343

Koen, C. 1998, MNRAS, 300, 567

Kostov, V. B., Moore, K., Tamayo, D., Jayawardhana, R., \& Rinehart, S. A. 2016, ApJ, 832, 183

Kupfer, T., Geier, S., Heber, U., et al. 2015, A\&A, 576, A44

Lenz, P. 2004, CoAst, 144, 41

Lutz, R. 2011, PhD thesis, Georg-August-Univ.

Lynas-Gray, A. E. 2013, in ASP Conf. Ser. 479, Progress in Physics of the Sun and Stars: A New Era in Helio- and Asteroseismology, ed. H. Shibahashi \& A. E. Lynas-Gray (San Francisco, CA: ASP), 273

Mullally, F., Winget, D. E., Degennaro, S., et al. 2008, ApJ, 676, 573

Németh, P., Kawka, A., \& Vennes, S. 2012, MNRAS, 427, 2180

O’Donoghue, D., Koen, C., \& Kilkenny, D. 1996, MNRAS, 278, 1075 
O’Donoghue, D., Lynas-Gray, A. E., Kilkenny, D., Stobie, R. S., \& Koen, C. 1997, MNRAS, 285, 657

Oreiro, R., Ulla, A., Pérez Hernández, F., et al. 2004, A\&A, 418, 243

Østensen, R., Oreiro, R., Solheim, J.-E., et al. 2010, A\&A, 513, 6

Østensen, R., Solheim, J.-E., Heber, U., et al. 2001, A\&A, 368, 175

Østensen, R. H., \& Van Winckel, H. 2012, in ASP Conf. Ser. 452, Fifth

Meeting on Hot Subdwarf Stars and Related Objects, ed. D. Kilkenny,

C. S. Jeffery, \& C. Koen (San Francisco, CA: ASP), 163

Otani, T. 2015, PhD thesis, Florida Institute of Technology

Paparo, M., Szeidl, B., \& Mahdy, H. A. 1988, Ap\&SS, 149, 73

Paxton, B., Bildsten, L., Dotter, A., et al. 2011, ApJS, 192, 3

Paxton, B., Cantiello, M., Arras, P., et al. 2013, ApJS, 208, 4

Paxton, B., Marchant, P., Schwab, J., et al. 2015, ApJS, 220, 15

Press, W. H., Teukolsky, S. A., Vetterling, W. T., \& Flannery, B. P. 1992, in Numerical Recipes (Cambridge: Cambridge Univ. Press), 251

Qian, S.-B., Zhu, L.-Y., Zola, S., et al. 2009, ApJ, 695, 163

Randall, S. K., Fontaine, G., Charpinet, S., et al. 2006, ApJ, 648, 637

Randall, S. K., Van Grootel, V., Fontaine, G., Charpinet, S., \& Brassard, P. 2009, A\&A, 507, 911

Saffer, R. A., Bergeron, P., Koester, D., \& Liebert, J. 1994, ApJ, 432, 531

Schechter, P. L., Mateo, M., \& Saha, A. 1993, PASP, 105, 1342

Schmidt-Kaler, T. 1982, Landolt-Bornstein New Series, Vol. 2b (New York: Springer)

Schuh, S., Huber, J., Dreizler, S., et al. 2006, A\&A, 445, 31

Silvotti, R., Østensen, R., Telting, J., \& Lovis, C. 2014, in ASP Conf. Ser. 481, 6th Meeting on Hot Subdwarf Stars and Related Objects, ed. V. Van Grootel et al. (San Francisco, CA: ASP), 13
Silvotti, R., Schuh, S., Janulis, R., et al. 2007, Natur, 449, 189

Silvotti, R., Szabó, R., Degroote, P., Østensen, R. H., \& Schuh, S. 2011, in AIP Conf. Proc. 1331, Planetary System Beyond the Main Sequence, ed. S. Schuh, H. Drechsel, \& U. Heber (College Park, MD: AIP), 133

Stobie, R. S., Kilkenny, D., O’Donoghue, D., et al. 1997, MNRAS, 287, 848

Sullivan, D. J., Metcalfe, T. S., O’Donoghue, D., Winget, D. E., et al. 2008, MNRAS, 387, 137

Tody, D. 1986, Proc. SPIE, 627, 733

Tody, D. 1993, in ASP Conf. Ser. 52, Astronomical Data Analysis Software and System II, ed. R. J. Hanisch, R. J. V. Brissenden, \& J. Barnes (San Francisco, CA: ASP), 173

Veras, D., Georgakarakos, N., Dobbs-Dixon, I., \& Gänsicke, B. T. 2017, MNRAS, 465, 2053

Völschow, M., Schleicher, D. R. G., Perdelwitz, V., \& Banerjee, R. 2016, A\&A, 587, A34

Vos, J., Németh, P., Vučković, M., Østensen, R., \& Parsons, S. 2017, MNRAS, 473,693

Vos, J., Østensen, R. H., Marchant, P., \& Van Winckel, H. 2015, A\&A, 579, A49

Wade, R., Barlow, B., Liss., S., \& Stark, M. 2014, in ASP Conf. Ser. 481, 6th Meeting on Hot Subdwarf Stars and Related Objects, ed. V. Van Grootel et al. (San Francisco, CA: ASP), 311

Winget, D. E., \& Kepler, S. O. 2008, ARA\&A, 46, 157

Xiong, H., Chen, X., Podsiadlowski, P., Li, Y., \& Han, Z. 2017, A\&A, 599, A54

Zorotovic, M., \& Schreiber, M. R. 2013, A\&A, 549, A95 